
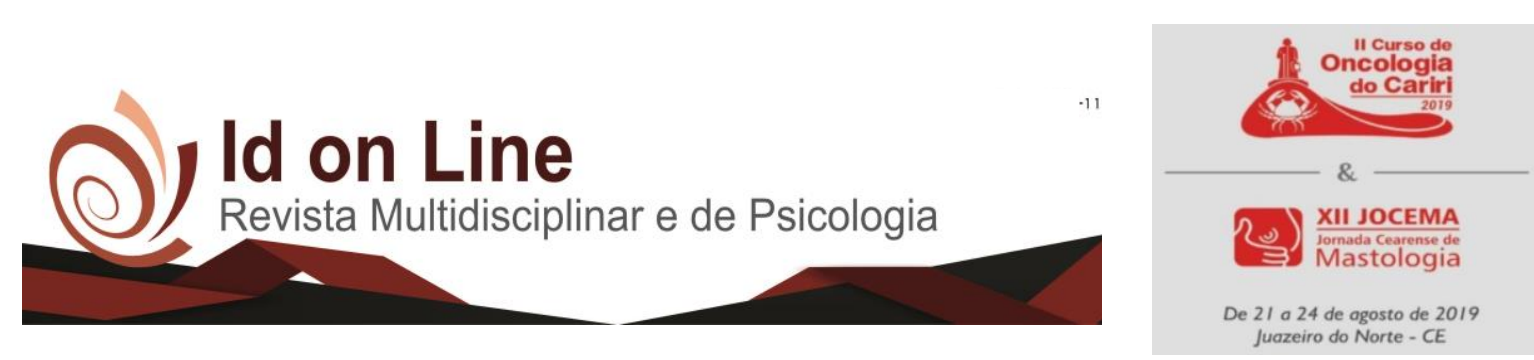

DOI: 10.14295/idonline.v13i46.1998

Resumo

\title{
O PAPEL DA OBESIDADE E DAS VARIAÇÕES DO ÍNDICE DE MASSA CORPORAL (IMC) AO LONGO DA VIDA ADULTA COMO FATOR DE RISCO PARA DESENVOLVIMENTO DE NEOPLASIA PANCREÁTICA
}

\author{
PINHO, Jonas Lima'; RAMIREZ, Fabian Danilo Unigarro'; SOUSA, Grecia Oliveiral ; \\ SOARES, Laryza Souza²; MORAIS, Letícia Bezerra ${ }^{3}$ NOBRE, Maria Elizabeth Pereira ${ }^{4}$
}

\begin{abstract}
Introdução: Apesar do câncer de pâncreas acometer somente $2 \%$ da população brasileira, estima-se que em 2030 se torne o segundo tipo de câncer mais frequente nos EUA. A ressecção cirúrgica é a única cura potencial para a maioria de carcinomas pancreáticos, mas, em $80 \%$ dos pacientes com sintomas, o tumor já é irressecável. É sabido que este tipo de câncer tem vários fatores que predispõem seu desenvolvimento, entre eles a obesidade. Objetivos: Avaliar o papel da obesidade e das variações do Índice de Massa Corporal (IMC) ao longo da vida adulta como fator de risco para desenvolvimento de neoplasia pancreática. Metodologia: $O$ presente trabalho consiste numa revisão sistemática norteada pela metodologia PRISMA, abordando estudos primários publicados na base de dados "Scopus (Elsevier)" entre 2016 e 2019 com os descritores: "cancer", "pancreas" e "obesity". Os critérios de inclusão foram: artigos publicados nos últimos 4 anos, em língua inglesa e hispânica que demonstrassem associação entre obesidade e risco de desenvolver neoplasias pancreáticas malignas. Foram excluídos comentários, revisões editoriais e cartas ao editor. Resultados: 101 estudos foram rastreados. Após leitura dos títulos e resumos e, posterior aplicação dos critérios de inclusão/exclusão, obtivemos uma amostra de 15 artigos. Estudos pontuam que homens e mulheres que eram obesos ou com excesso de peso, quando adolescentes, estão em um risco aumentado para posterior câncer pancreático. Outros estudos afirmam que as trajetórias do IMC caracterizadas pelo sobrepeso no início da idade adulta foram associadas ao aumento do risco de câncer no pâncreas, sugerindo uma abordagem ao risco de doenças em toda a vida. $\mathrm{O}$ tecido adiposo sintetiza e segrega muitos fatores de crescimento e várias citocinas conhecidas como adipocinas, tais como a leptina. Pesquisas demonstram que há uma correlação significativa entre baixos níveis de leptina e câncer pancreático. Entretanto, é importante salientar que o desenvolvimento de câncer pancreático está relacionado a outros fatores que não necessariamente estejam ligados ao IMC durante a vida, de modo que variáveis como nutrição, prática de ativiades físicas e o próprio metabolismo também devem ser consideradas. Conclusão: É importante o controle do IMC ao longo da vida, pois através deste método pode-se prevenir comorbidades como a obesidade que, em última instância, podem aumentar a incidência de neoplasias pancreáticas na população brasileira.
\end{abstract}

Palavras-chave: câncer, pâncreas, obesidade, IMC, leptina.

\footnotetext{
${ }^{1}$ Acadêmico(a) do curso de Medicina da Universidade Federal do Cariri (UFCA), Barbalha, CE. jonaslpinho@ gmail.com, fabiunir@hotmail.es, grecia.oliveirasousa@gmail.com,

${ }^{2}$ Acadêmica da Faculdade de Medicina Estácio de Juazeiro do Norte (FMJ). laryzasouza@ gmail.com;

${ }^{3}$ Acadêmica do curso de Medicina da Faculdade Santa Maria (FSM), Cajazeiras - PB. leticia.bmorais1 @ gmail.com;

${ }^{4}$ Professora Adjunta do curso de Medicina da Universidade Federal do Cariri (UFCA), Barbalha - CE. elizabeth.nobre@ufca.edu.br.
} 


\section{Referências}

BABIC, Ana et al. Pancreatic Cancer Risk Associated with Prediagnostic Plasma Levels of Leptin and Leptin Receptor Genetic Polymorphisms. Cancer Research, [s.1.], v. 76, n. 24, p.7160-7167, 25 out. 2016. American Association for Cancer Research (AACR).

CARBONE, $\mathrm{C}$ et al. Adipocytes sustain pancreatic cancer progression through a non-canonical WNT paracrine network inducing ROR2 nuclear shuttling. International Journal Of Obesity, [s.1.], v. 42, n. 3, p.334-343, 20 nov. 2017. Springer Science and Business Media LLC.

CHOI, e $\mathrm{K}$ et al. Body mass index and 20 specific cancers: re-analyses of dose-response metaanalyses of observational studies. Annals Of Oncology, [s.1.], v. 29, n. 3, p.749-757, 28 dez. 2017. Oxford University Press (OUP).

FREISLING, Heinz et al. Comparison of general obesity and measures of body fat distribution in older adults in relation to cancer risk: meta-analysis of individual participant data of seven prospective cohorts in Europe. British Journal Of Cancer, [s.1.], v. 116, n. 11, p.1486-1497, 25 abr. 2017. Springer Nature.

KADRI COLAKOGLU, M. et al. Roles of adiponectin and leptin as diagnostic markers in pancreatic cancer. Bratislava Medical Journal, [s.1.], v. 118, n. 07, p.394-398, 2017. AEPress, s.r.o.

LEVI, Zohar et al. Adolescent overweight and obesity and the risk for pancreatic cancer among men and women: a nationwide study of 1.79 million Israeli adolescents. Cancer, [s.1.], v. 125, n. 1, p.118-126, 12 nov. 2018. Wiley.

RUBEIS, Vanessa de et al. Trajectories of body mass index, from adolescence to older adulthood, and pancreatic cancer risk; a population-based case-control study in Ontario, Canada. Cancer Causes \& Control, [s.1.], v. 30, n. 9, p.955-966, 22 jun. 2019. Springer Science and Business Media LLC.

SOLDAN, MÔnica. Pancreatic cancer screening. Revista do Colégio Brasileiro de Cirurgiões, [s.1.], v. 44, n. 2, p.109-111, abr. 2017. FapUNIFESP (SciELO).

TANG, Xiaodong et al. The role of the fat mass and obesity-associated protein in the proliferation of pancreatic cancer cells. Oncology Letters, [s.1.], p.2473-2478, 28 dez. 2018. Spandidos Publications. 